\title{
LETTER TO THE EDITOR \\ Cervical spinal cord injuries without radiographic evidence of trauma: a prospective study
}

Spinal Cord (2014) 52, 84; doi:10.1038/sc.2013.141; published online 19 November 2013

The study by Mohanty et al. ${ }^{1}$ correlates magnetic resonance imaging (MRI) findings and initial neurological status with the mid-term outcome of patients presenting with discordant clinical and radiological findings after cervical spinal cord trauma. They enrolled 45 patients and employed a standardized diagnostic and conservative treatment protocol. Their detailed description of the largest nonoperatively managed cohort of patients with spinal cord injuries without radiographic evidence of trauma (SCIWORET) is an important contribution to the field.

Observed in $16.5 \%$ of all cervical spine injuries, the relatively high incidence rate of SCIWORET is especially noteworthy. Their findings suggest a correlation between initial neurologic status and mid-term functional outcome, and describe a statistically significant correlation between intraspinal MRI findings and initial as well as mid-term neurologic status. Finally, Mohanty et al. ${ }^{1}$ highlight the importance of the length of prevertebral hyperintensity (PVH) as a risk factor for poor neurological outcome, thus paralleling the results of a previously published operatively managed cohort.

However, our recent systematic review of spinal cord injuries without radiographic abnormality (SCIWORA) and SCIWORA-like conditions (for example, SCIWORET, SCIWOCTET) identified the lack of a conclusive classification system of MRI findings for patients presenting with a clinico-radiological mismatch after cervical spinal cord trauma. ${ }^{2}$

In 1997, Ramon et al. ${ }^{3}$ expanded a systematic approach for the description of intramedullary findings on MRI, which was adopted and modified by many authors including Mohanty et al., ${ }^{1}$ who added 'medullary swelling', thus impairing comparability between the individual studies. Furthermore, the authors limited their report of extramedullary findings to $\mathrm{PVH}$, thereby ignoring the importance of other extramedullary MRI abnormalities.

Taken together, the conclusions of Mohanty et al. ${ }^{1}$ are supported by our current comprehensive review, yet we recommend the use of a standardized MRI classification that incorporates extramedullary as well as intramedullary findings for future studies.

\section{CONFLICT OF INTEREST}

The authors declare no conflict of interest.

CK Boese ${ }^{1}$ and P Lechler ${ }^{2}$ ${ }^{1}$ Department of Orthopaedic and Trauma Surgery, University Hospital of Cologne, Cologne, Germany and ${ }^{2}$ Department of Trauma, Hand and Reconstructive Surgery, University of Giessen and Marburg, Marburg, Germany E-mail: christoph.boese@gmail.com

1 Mohanty SP, Bhat NS, Singh KA, Bhushan M. Cervical spinal cord injuries without radiographic evidence of trauma: a prospective study. Spinal Cord 2013; 51 815-818.

2 Ramon S, Dominguez R, Ramirez L, Paraira M, Olona M, Castello T. Clinical and magnetic resonance imaging correlation in acute spinal cord injury. Spinal Cord 1997 35: 664-673.

3 Boese CK, Lechler P. Spinal cord injury without radiologic abnormalities in adults: a systematic review. J Trauma Acute Care Surgery 2013; 75: 320-330. 